\title{
Photoluminescence intermittency in an individual single-walled carbon nanotube at room temperature
}

\section{$\operatorname{AUTHOR}(\mathrm{S}):$}

Matsuda, K.; Kanemitsu, Yoshihiko; Irie, K.; Saiki, T.; Someya, T.; Miyauchi, Y.; Maruyama, S.

\section{CITATION:}

Matsuda, K....[et al]. Photoluminescence intermittency in an individual single-walled carbon nanotube at room temperature. Applied Physics Letters 2005, 86(12): 123116.

\section{ISSUE DATE:}

2005-03

URL:

http://hdl.handle.net/2433/87366

RIGHT:

c 2005 American Institute of Physics. 


\title{
Photoluminescence intermittency in an individual single-walled carbon nanotube at room temperature
}

\author{
K. Matsuda $a^{a, b), c)}$ and Y. Kanemitsu \\ International Research Center for Elements Science, Institute for Chemical Research, Kyoto University, Uji, \\ Kyoto 611-0011, Japan \\ K. Irie and T. Saiki ${ }^{\mathrm{a})}$ \\ Department of Electronics and Electrical Engineering, Keio University, 3-14-1 Hiyoshi, Kohoku, Yokohama, \\ Kanagawa 223-8522, Japan \\ T. Someya \\ Department of Applied Physics, The University of Tokyo, 7-3-1 Hongo, Bunkyo, Tokyo 113-8656, Japan
}

Y. Miyauchi and S. Maruyama

Department of Mechanical Engineering, The University of Tokyo, 7-3-1 Hongo, Bunkyo, Tokyo 113-8656, Japan

(Received 27 September 2004; accepted 8 February 2005; published online 18 March 2005)

\begin{abstract}
We described the photoluminescence (PL) properties of individual micelle-encapsulated single-walled carbon nanotubes (SWNTs) at room temperature. Single PL peak from isolated individual SWNT with a chiral index of $(6,5)$ showed a linear increase and saturation behavior of the PL intensity. Unusual PL intensity fluctuation in the temporal evolutions of the PL intensity, referred to as PL intermittency, was seen with some SWNTs, while the PL intensity with most SWNTs remained at a constant amplitude. The mechanism of the PL intermittency was discussed.
\end{abstract}

(C) 2005 American Institute of Physics. [DOI: 10.1063/1.1894609]

Single-walled carbon nanotubes (SWNTs) have attracted a great deal of attention because of their potential use in electronic devices and their unique physical properties. ${ }^{1-3}$ Recently, photoluminescence (PL) signals were observed from micelle-encapsulated SWNTs ${ }^{4,5}$ and SWNTs suspended between pillars above a silicon substrate ${ }^{6,7}$ when the SWNTs were prevented from becoming bundled or contacting the substrate. PL spectroscopy has revealed new insights into the optical and electronic properties of SWNTs. ${ }^{4-9}$ Bachilo et al. demonstrated that the chirality of SWNTs can be determined from the energy positions in the PL spectra and PL excitation spectra. ${ }^{5}$ However, SWNTs are an inhomogeneous system because the optical transition energy differs from a SWNT even for those with the same chirality. ${ }^{10,11}$ As a result, the macroscopic PL spectrum reflects the ensemble average of signals from many SWNTs and is inhomogeneously broadened. This makes it difficult to investigate the intrinsic optical properties of a SWNT, such as the homogeneous linewidth or variation in the PL intensity from a SWNT to a SWNT. Spectroscopic observation of an individual SWNT, called individual SWNT spectroscopy, is useful for understanding the physics of the inhomogeneous system, ${ }^{10-12}$ as with the spectroscopy of fluorescent dye molecules ${ }^{13}$ and semiconductor quantum dots. ${ }^{14}$ Single molecule spectroscopy enabled us to reveal the intrinsic homogeneous linewidth and unusual phenomena, such as fluorescence (photoluminescence) blinking or intermittency, ${ }^{13}$ in which the emission peak and intensity fluctuate over time.

\footnotetext{
a) Also at Kanagawa Academy of Science and Technology, 3-2-1 Sakado, Takatsu, Kawasaki 213-0012, Japan.

${ }^{b)}$ Also at Nanostructure and Material Property, PRESTO, Japan Science and Technology Agency, 4-1-8 Honcho Kawaguchi, Saitama 332-0012, Japan.

${ }^{c)}$ Electronic mail: matsuda@scl.kyoto-u.ac.jp
}

In this letter, we report on the PL spectrum and time traces of the PL intensity of individual micelle-encapsulated SWNTs at room temperature. We found clear differences in the time traces from a SWNT to a SWNT, i.e., most SWNTs showed stable emissions, although PL intermittency behavior was seen in specific SWNTs. The room temperature emission properties of an individual SWNT will provide us with important information for fabricating optical devices at the single SWNT level. ${ }^{15}$

A zeolite-supported metal catalyst was prepared using a reported procedure. ${ }^{16,17}$ Cobalt acetate and iron acetate were impregnated into USY-zeolite powders. The amounts of Co and Fe were $2.5 \mathrm{wt} \%$ each, with respect to the zeolite powder. The details of the alcohol catalytic chemical vapor deposition (ACCVD) procedure have been reported. ${ }^{17}$ The catalysts were placed in a quartz boat, which was set in a quartz tube inside an electric furnace. While heating of the electric furnace, $\mathrm{Ar} / \mathrm{H}_{2}\left(3 \% \mathrm{H}_{2}\right)$ was supplied at $300 \mathrm{sccm}$ to maintain the pressure inside the quartz tube at $300 \pm 20$ Torr. After the electric furnace reached the growth temperature of $650{ }^{\circ} \mathrm{C}$, the $\mathrm{Ar} / \mathrm{H}_{2}$ was stopped, and the quartz tube was evacuated using a rotary pump. Vapor from an ethanol reservoir was then introduced for $10 \mathrm{~min}$ at a constant pressure of 10 Torr. After the CVD reaction, the furnace was turned off, and $100 \mathrm{sccm} \mathrm{Ar} / \mathrm{H}_{2}$ was allowed to flow through the tube while it cooled to room temperature.

The samples investigated in this study were SWNTs in a surfactant suspension. These were prepared following a procedure similar to Ref. 4 . The SWNTs were dispersed in $\mathrm{D}_{2} \mathrm{O}$ with $1 \mathrm{wt} \%$ sodium dodecyl sulfate (SDS) by sonication with an ultrasonic processor for $1 \mathrm{~h}$. This suspension was then centrifuged for $24 \mathrm{~h}$ at $20627 \mathrm{~g}$. The SWNTs synthesized were characterized using resonant Raman scattering and macroscopic PL spectroscopy. ${ }^{18}$ The SWNTs were dispersed and dried on a glass slip using a spin-coating method. 


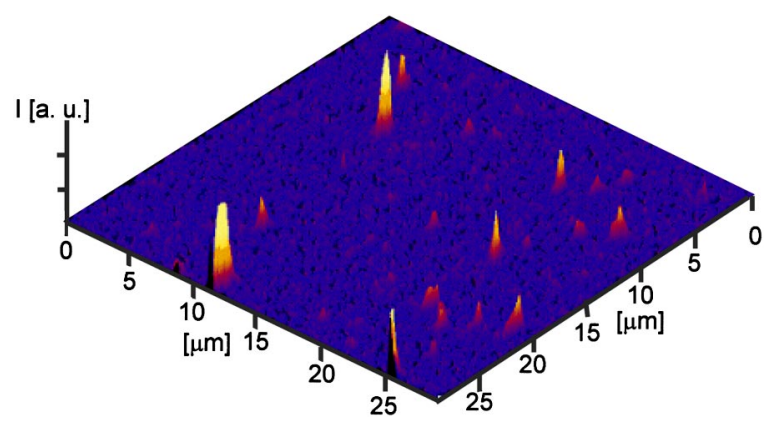

FIG. 1. (Color online) Three-dimensional map of the PL intensity of individual SWNTs at room temperature, excited at $2.33 \mathrm{eV}$. The scanning area of the image is $28 \mu \mathrm{m} \times 28 \mu \mathrm{m}$.

Spectroscopic studies of individual SWNTs were carried out at room temperature using a home-built scanning confocal microscope. The SWNTs on the scanning stage were illuminated with the focused spot of a linearly polarized laser diode light $(\lambda=532 \mathrm{~nm})$ and $\mathrm{He}-\mathrm{Ne}$ laser light $(\lambda$ $=632.8 \mathrm{~nm}$ ) through an oil immersion objective (numerical aperture 1.25). PL signals from individual SWNTs were detected using a $\mathrm{Si}$ avalanche photodiode to acquire PL images. PL spectra were obtained using a $32 \mathrm{~cm}$ monochromator equipped with a cooled charge coupled device (CCD).

Figure 1 shows three-dimensional plot of a SWNT PL image, detected at a range of $1.18-1.37 \mathrm{eV}$ at room temperature. Each bright spot corresponds to the PL signal from an individual SWNT, as confirmed by the PL spectrum, which had a single PL peak (described below in detail). The variation in the intensities of the PL image results from the difference in the emission wavelengths of the SWNTs, which depend on chirality, as the detection efficiency strongly decreases toward the lower energy side. The random orientation of the SWNTs on the substrate also contributes to the variation in the PL intensity, because the PL intensity has strong polarization dependence on the polarization direction of the excitation light with relative to a SWNT. ${ }^{10,12}$

Figure 2(a) shows a macroscopic PL spectrum of micelle-encapsulated SWNTs in $\mathrm{D}_{2} \mathrm{O}$, obtained at $1.95 \mathrm{eV}$ excitation condition (dotted line). The PL spectrum is com-

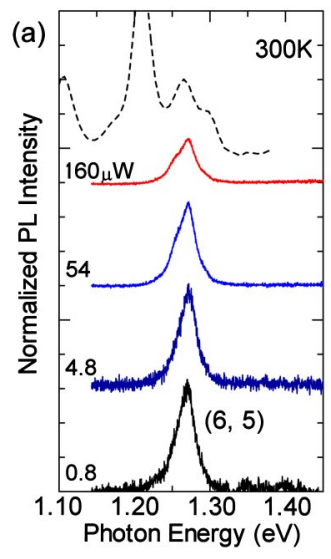

(b)

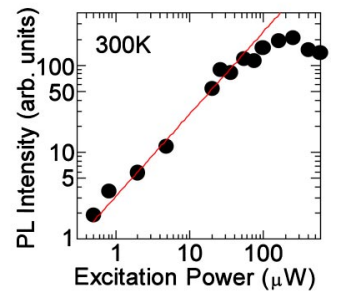

FIG. 2. (Color online) (a) Macroscopic PL spectrum of micelleencapsulated SWNTs in $\mathrm{D}_{2} \mathrm{O}$ at the excitation photon energy of $1.95 \mathrm{eV}$ (dotted line). PL spectra of an individual SWNT with a chirality of $(6,5)$ at the excitation photon energy of $1.96 \mathrm{eV}$ under various excitation powers (solid lines). Each PL spectrum is normalized using the excitation power; (b) Excitation power dependence of the PL intensity. The solid line corresponds to the gradient associated with linear power dependence.

Downloaded 16 Nov 2009 to 130.54.110.32. Redistribution subject (a)

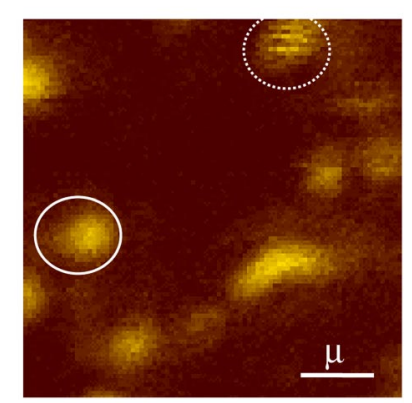

(b)

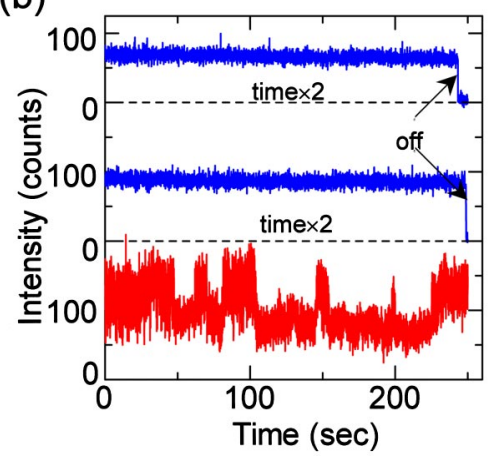

FIG. 3. (Color online) (a) PL image of individual SWNTs at the excitation photon energy of $1.96 \mathrm{eV}$ in a $5.1 \mu \mathrm{m} \times 5.1 \mu \mathrm{m}$ area; (b) typical temporal evolution of the PL intensities of individual SWNTs showing stable emission (blue lines)(upper and middle panels). Time trace of the PL intensities of a specific SWNT showing PL intermittency (red line) (lower panel). The excitation laser was blocked in the "off" region.

posed of several peaks from SWNTs with various chiralities; the peaks are assigned as $(8,3),(6,5),(7,5)$, and $(7,6)$ from the higher to lower energy side. ${ }^{5,18}$ The solid lines in Fig. 2(a) show an example of the PL spectra of an individual SWNT, obtained by varying the excitation power. Each PL spectrum is normalized by the excitation power. The PL spectra show a single peak, in contrast to the various peaks seen in the macroscopic PL spectrum. The spectral position of the peak from an individual SWNT in the PL spectrum does not change with the excitation power, indicating that the many body effects such as band-gap renormalization do not occur in this excitation regime using continuous wave laser light. We plotted the PL intensity as a function of the excitation power in Fig. 2(b). The PL intensity (closed circles) increased linearly before gradually saturating at higher excitation conditions. These experimental results imply that the emission peak originates from recombination between the lowest band edge state $\left(E_{11}\right)$.

Next, we investigated time traces of PL intensity from individual SWNTs. Figure 3(a) shows an expanded PL image on a $5.1 \mu \mathrm{m} \times 5.1 \mu \mathrm{m}$ scale. We observed that most of the PL signals in the PL image (e.g., the solid line circled area) are smooth spots, and the time traces of the PL signals in Fig. 3(b) (blue lines) (upper and middle panels) show constant emission behavior, as reported previously. ${ }^{10}$ By contrast, a few PL spots with scratch noises are observed (e.g., within the dotted circle). The noise in the spot is caused by fluctuation in the PL intensity. As shown in the time trace in Fig. 3(b) (red line) (lower panel), the PL intensity switches between high and low emission states. This phenomenon is referred to as fluorescence (photoluminescence) intermittency, in which the PL intensity switches multiple levels as time passes. ${ }^{19-21}$ Thermal heating by the excitation laser was time passes.
AIP license or copyright; see http://apl.aip.org/apl/copyright.jsp 
ruled out as the origin of this PL intensity fluctuation, because we acquired the data at lower excitation power. Furthermore, if thermal heating caused the PL intensity fluctuation, the PL intensity fluctuation should be observed in all SWNTs.

PL intermittency and blinking are observed frequently in single dye molecules ${ }^{13,19}$ and single semiconductor quantum dots. ${ }^{14,20,21}$ Several mechanisms have been proposed to explain these phenomena, ${ }^{13,14,19-21}$ because the time scale of the PL intermittency and on-off blinking phenomena depend on the system and experimental conditions. As a possible mechanism, the reorientation of a molecule (direction of the dipole moment) could cause the PL intensity fluctuation; however, the SWNTs with 100-300 nm length are fixed on the substrate surface by strong van der Waals interactions. Therefore, the observed PL intermittency is unlikely to be the result of the reorientation of SWNTs on the substrate. The fluctuation in the local environment perturbs the electronic states of SWNTs and hence the PL intensity. In the SWNT showing the PL intermittency, there are localized states owing to chemical or structural defects in the SDS or the SWNT itself. ${ }^{22,23}$ When a charge is trapped by such a localized state, the charge results in a strong, local electric field $^{21}$ on the SWNT. As a result, the overlap of the wave functions of an electron and a hole in the SWNT is decreased, reducing the PL intensity. The trapped charge is annihilated by recombination, and the local electric field caused by the trapped carrier disappears. This cycle (the creation and annihilation of localized charges) might induce the PL intermittency in SWNTs on the time scale observed.

In summary, we presented PL spectra and showed the temporal evolution of the PL intensity in an individual SWNT in a surfactant at room temperature. The PL intensity of a single PL peak from an individual SWNT shows a linear dependence on the excitation power, and the energy position does not change with higher excitation. The amplitude of the PL intensity in most SWNTs remains constant over time. Nevertheless, in some SWNTs, the PL intensity fluctuates. The fluctuation in the local electric field causes the PL intermittency in a SWNT. We obtained emission properties of an individual SWNT at room temperature including these variations from a SWNT to a SWNT, which should provide us with important insights for the fabrication of optical devices at the single SWNT level.
This study was performed as a part of the Active NanoCharacterization and Technology Project, supported by Special Coordination Funds from the Ministry of Education, Culture, Sports, Science, and Technology of Japan.

${ }^{1}$ S. Iijima and T. Ichihashi, Nature (London) 363, 603 (1993).

${ }^{2}$ R. Saito, G. Dresselhaus, and M. S. Dresselhaus, Physical Properties of Carbon Nanotubes (World Scientific, Singapore, 1998).

${ }^{3}$ M. S. Dresselhaus, G. Dresselhaus, and P. Avouris, Carbon Nanotubes (Springer, Berlin, 2001).

${ }^{4}$ M. J. O'Connell, S. M. Bachilo, C. B. Huffman, V. C. Moore, M. S. Strano, E. H. Haroz, K. L. Rialon, P. J. Boul, W. H. Noon, C. Kittrell, J. Ma, R. H. Hauge, R. B. Weisman, and R. E. Smalley, Science 297, 593 (2002).

${ }^{5}$ S. M. Bachilo, M. S. Strano, C. Kittrell, R. H. Hauge, R. E. Smalley, and R. B. Weisman, Science 298, 2361 (2002).

${ }^{6}$ J. Lefebvre, Y. Homma, and P. Finnie, Phys. Rev. Lett. 90, 217401 (2003).

${ }^{7}$ J. Lefebvre, J. M. Fraser, Y. Homma, and P. Finnie, Appl. Phys. A: Mater. Sci. Process. 78, 1107 (2004).

${ }^{8}$ S. Lebedkin, F. Hennrich, T. Skipa, and M. Kappes, J. Phys. Chem. B 107, 1945 (2003).

${ }^{9}$ S. Lebedkin, K. Arnold, F. Hennrich, R. Krupke, B. Renker, and M. Kappes, New J. Phys. 5, 140 (2003).

${ }^{10}$ A. Hartschuh, H. N. Pedrosa, L. Novotny, and T. D. Krauss, Science 301, 1354 (2003).

${ }^{11}$ H. Htoon, M. J. O'Connell, P. J. Cox, S. K. Doorn, and V. I. Klimov, Phys. Rev. Lett. 93, 027401 (2004).

${ }^{12}$ J. Lefebvre, J. M. Fraser, P. Finnie, and Y. Homma, Phys. Rev. B 69, 075403 (2004).

${ }^{13}$ H. P. Lu and X. S. Xie, Nature (London) 385, 143 (1997).

${ }^{14}$ M. Nirmal, B. O. Dabbousi, M. G. Bawendi, J. J. Macklin, J. K. Trautman, T. D. Harris, and L. E. Brus, Nature (London) 383, 802 (1996).

${ }^{15}$ J. A. Misewich, R. Martel, P. Avouris, J. C. Tsang, S. Heinze, and J. Tersoff, Science 300, 783 (2003).

${ }^{16}$ S. Maruyama, R. Kojima, Y. Miyauchi, S. Chiashi, and M. Kohno, Chem. Phys. Lett. 360, 229 (2002).

${ }^{17}$ Y. Murakami, Y. Miyauchi, S. Chiashi, and S. Maruyama, Chem. Phys. Lett. 374, 53 (2003).

${ }^{18}$ Y. Miyauchi, S. Chiashi, Y. Murakami, Y. Hayashida, and S. Maruyama, Chem. Phys. Lett. 387, 198 (2004).

${ }^{19}$ R. M. Dickson, A. B. Cubitt, R. Y. Tsien, and W. E. Moerner, Nature (London) 388, 355 (1997).

${ }^{20}$ S. A. Empedocles and M. G. Bawendi, Science 278, 2114 (1997).

${ }^{21}$ M. Sugisaki, H.-W. Ren, K. Nishi, and Y. Masumoto, Phys. Rev. Lett. 86, 4883 (2001).

${ }^{22}$ T. W. Tombler, C. Zhou, L. Alexseyev, J. Kong, H. Dai, L. Lei, C. S. Jayanthi, M. Tang, and S. Wu, Nature (London) 405, 769 (2000).

${ }^{23}$ P. L. McEuen, M. Bockrath, D. H. Cobden, Y. G. Yoon, and S. G. Louie, Phys. Rev. Lett. 83, 5098 (1999). 